\title{
Prospective phase II study evaluating the efficacy of swallow ability screening tests and pneumonia prevention using a team approach for elderly patients with gastric cancer
}

\author{
Yuichiro Miki $^{1,2} \cdot$ Rie Makuuchi $^{1} \cdot$ Shinsaku Honda $^{1} \cdot$ Masanori Tokunaga $^{1} \cdot$ \\ Yutaka Tanizawa $^{1}$ - Etsuro Bando ${ }^{1}$ - Taiichi Kawamura ${ }^{1}$ - Takashi Yurikusa ${ }^{3}$. \\ Akira Tanuma $^{4} \cdot$ Masanori Terashima $^{1}$ (D)
}

Received: 23 March 2017/Accepted: 2 June 2017/Published online: 13 June 2017

(c) The International Gastric Cancer Association and The Japanese Gastric Cancer Association 2017

\begin{abstract}
Background Aging partly impairs swallowing function, which is considered a risk factor for postoperative pneumonia (PP). We evaluated the efficacy of a new team-based strategy to reduce the incidence of PP in elderly patients with gastric cancer.

Methods This single-center, prospective phase II study included elderly patients ( $\geq 75$ years old) with gastric cancer undergoing gastric surgery. The primary endpoint was the incidence of Clavien-Dindo grade II or higher PP. Patients were initially screened using three swallowing function screening tests: a symptom questionnaire, the modified water swallow test (MSWT), and the repetitive saliva swallowing test (RSST). All patients were provided standard preoperative oral checks and care and simple neck muscle training. For patients who screened positive, a videofluorographic swallowing study was performed; if an abnormality was found, the patient was given intensive swallowing rehabilitation both pre- and postoperatively.
\end{abstract}

Electronic supplementary material The online version of this article (doi:10.1007/s10120-017-0736-3) contains supplementary material, which is available to authorized users.

Masanori Terashima

m.terashima@scchr.jp

1 Division of Gastric Surgery, Shizuoka Cancer Center, 1007 Shimonagakubo, Nagaizumi-Cho, Shizuoka 411-8777, Japan

2 Department of Surgical Oncology, Osaka City University Graduate School of Medicine, Osaka, Japan

3 Division of Dentistry and Oral Surgery, Shizuoka Cancer Center, Shizuoka, Japan

4 Division of Rehabilitation Medicine, Shizuoka Cancer Center, Shizuoka, Japan
Results Of 86 eligible patients enrolled, PP developed in 3 (3.5\%). The $60 \%$ confidence interval of $1.8-6.3 \%$ had an upper limit below the prespecified threshold of $7.8 \%$. Positive screening results were found for 19 patients $(22.1 \%)$ on the symptom questionnaire, $3(3.5 \%)$ on the MSWT, and $1(1.2 \%)$ on the RSST. PP was not observed in any patients who screened positive.

Conclusion In conclusion, although the screening tests we adopted here were not sufficient to identify patients at high risk of aspiration pneumonia, perioperative interventions using a team approach might be effective in reducing the incidence of PP in elderly patients with gastric cancer.

Keywords Gastric cancer · Elderly patients · Pneumonia

\section{Introduction}

Gastric cancer is the second leading cause of death among all malignant neoplasms [1,2]. For patients with locally advanced gastric cancer, gastrectomy with lymphadenectomy is currently the only potentially curative treatment. As a result of population aging, an increasing number of elderly patients with gastric cancer undergo surgery. According to the Cancer Registries for the Monitoring of Cancer Incidence in Japan, the number of patients aged $\geq 75$ years with gastric cancer increased from $18.3 \%$ in 1970 to $43.3 \%$ in 2010 [3]. The incidence of postoperative complications remains high in elderly patients, because such patients tend to have impaired physiologic function of various organs as well as concurrent diseases [4]. In particular, aging-related anatomical and physiologic changes tend to impair swallowing in elderly people, which increases the risk of postoperative pneumonia [3, 5-7]. We previously reported that for patients aged 75 years or older, 
swallowing dysfunction was an independent risk factor for postoperative pneumonia after gastrectomy [8].

As a result of our previous study, in 2015 we planned a phase II study to evaluate a new program to reduce the incidence of postoperative pneumonia in elderly patients with gastric cancer, based on preoperative screening and intervention for those at risk. For initial screening, we used three swallowing function screening tests. If the screening results were negative, the patients underwent an oral check and care performed by dentists and dental hygienists as well as simple neck muscle training preoperatively. If the screening results were positive, a videofluorographic swallowing study (VFSS) was performed. For patients whose VFSS showed abnormalities, intensive pre- and postoperative swallowing rehabilitation was provided. Each of these steps have been used in the perioperative management of patients with esophageal or head and neck cancer, but this team-based strategy has not been specifically investigated in elderly patients undergoing surgery for gastric cancer. Therefore, the aim of this single-center prospective phase II study was to assess the effectiveness of a team-based swallow ability screening and intervention program to reduce the incidence of postoperative pneumonia (ClavienDindo classification grade II or higher) in elderly patients with gastric cancer.

\section{Patients and methods}

\section{Patients}

This study was designed as a single-center, prospective phase II trial. The Institutional Review Board of Shizuoka Cancer Center approved the study protocol. Inclusion criteria were as follows: histologically confirmed adenocarcinoma of the stomach, age 75 years or older, adequate oral intake, an Eastern Cooperative Oncology Group performance status of $0-1$, no prior chemotherapy or radiotherapy for any malignancy, adequate organ function, and written informed consent. The study was registered with the University Hospital Medical Information Network (UMIN000025541; http://www.umin.ac.jp/ctr/).

\section{Swallow ability screening tests}

Three screening tests assessing for a deglutition disorder were used: a symptom questionnaire, the repetitive saliva swallowing test (RSST), and the modified water swallow test (MWST). We adopted these tests from the treatment guidelines for deglutition disorders, which were published by The Oto-Rhino-Laryngological Society of Japan [9].

The symptom questionnaire is shown in Table 1 .
For the RSST, the patient was asked to swallow saliva repeatedly. A physician confirmed laryngeal elevation with the swallowing reflex and counted the frequency of voluntary swallows within $30 \mathrm{~s}$ [6].

For the MWST, we asked patients to drink $30 \mathrm{ml}$ of water [10]. We evaluated the presence or absence of the swallowing reflex, dyspnea, cough, and wet hoarseness. The swallowing profiles based on this test are shown in Table 2.

\section{Rehabilitation program}

Stretching of the neck muscles and simple respiratory training with incentive spirometry were used to prevent postoperative pneumonia before 2015 .

Exercise aimed at the suprahyoid upper esophageal sphincter (UES) opening muscles has been shown to be effective for decreasing the resistance to bolus flow by increasing the deglutitive UES opening [11]. Thus, we asked all participants to do the following exercise preoperatively. They were instructed to sit and place a hand on their forehead. They were then told to push their head forward against the backward pressure of the hand. This move was sustained for $5 \mathrm{~s}$, followed by five consecutive repetitions of the head movement in the same position for $1 \mathrm{~s}$ each. Participants were instructed to perform this entire exercise 3-5 times per day.

If a screening test was positive, we referred these patients to the division of rehabilitation medicine. A physician from this division examined these patients and performed VFSS. If VFSS results were abnormal, the rehabilitation team prepared an intensive individualized rehabilitation treatment program for the patient. The following measures were used as indicated by the individual's condition (Supplementary Fig. 1).

1. Exercising the oral cavity.

2. Voice projection training.

3. "Ice massage" in which we attach a piece of ice to the end of a stick and apply it to the pharynx.

4. "Pushing exercise" to correct glottal incompetence.

5. Advising patients on how to eat (the amount of food in a mouthful, neck position while eating, etc.).

6. Thickening fluids when oral intake was resumed after the operation.

7. Observing how patients eat, repeating tests to assess swallowing function, and deciding if patients need to continue consuming thickened fluids.

\section{Oral care program}

The oral care program was performed for all participants by the dental team during the perioperative period. Before 
Table 1 Symptom questionnaire to evaluate swallow ability

\begin{tabular}{|c|c|c|c|}
\hline (1) Have you ever been diagnosed with pneumonia? & A. More than twice & B. Once & C. Never \\
\hline (2) Have you recently experienced any weight loss? & A. Yes & B. Slight & C. None \\
\hline (3) Have you had any trouble swallowing? & A. Frequently & B. Sometimes & C. Never \\
\hline (4) Have you coughed up anything after eating? & A. Frequently & B. Sometimes & C. Never \\
\hline (5) Have you coughed up anything after drinking? & A. Frequently & B. Sometimes & C. Never \\
\hline (6) Have you felt any tickling in the throat? & A. Frequently & B. Sometimes & C. Never \\
\hline (7) Have you ever felt like you have any food left in your throat after swallowing? & A. Frequently & B. Sometimes & C. Never \\
\hline (8) Has your pace of eating slowed? & A. Frequently & B. Sometimes & C. Never \\
\hline (9) Have you had any trouble eating solid food? & A. Frequently & B. Sometimes & C. Never \\
\hline (10) Has any food involuntarily fallen out of your mouth while eating? & A. Frequently & B. Sometimes & C. Never \\
\hline (11) Have you ever felt like you have any food left in your mouth after eating? & A. Frequently & B. Sometimes & C. Never \\
\hline (12) Have you felt any food or stomach acid in the throat? & A. Frequently & B. Sometimes & C. Never \\
\hline (13) Have you had any trouble sleeping in the middle of night because of your cough? & A. Frequently & B. Sometimes & C. Never \\
\hline (14) Have you recently lost your voice? & A. Frequently & B. Sometimes & C. Never \\
\hline
\end{tabular}

Patients who answered "A" to any of these questions were considered to have abnormal swallow ability. Patients who answered "B" to any of these questions were suspected to have abnormal swallow ability

Table 2 Evaluation of modified water swallow test

\begin{tabular}{ll}
\hline Profile & \\
\hline 1 & Can drink at once without coughing up \\
2 & Can drink in two or more swallows without coughing up \\
3 & Can drink at once but cough up \\
4 & Drinks in two or more swallows and coughs up \\
5 & Coughs up many times and cannot drink all $30 \mathrm{ml}$ \\
\hline
\end{tabular}

Patients with profile 3,4 , or 5 were considered to have abnormal swallow ability

surgery, patients received routine oral screening by dentists to determine the status of their teeth, periodontal tissue, and oral hygiene. Professional oral health care, including mechanical tooth cleaning, was provided by dental hygienists. In addition, any required dental treatment was performed. To maintain good oral hygiene, patients were given instructions about tooth brushing, oral mucosa cleaning, and denture care.

\section{Definition and severity of pneumonia}

Postoperative pneumonia was defined as an infection of one or both lungs diagnosed by radiologic criteria and clinical findings within 30 days after surgery. The radiologic criteria included a definitive chest radiologic examination (X-ray or CT) demonstrating at least one of the following: (1) new or progressive and persistent infiltrate, (2) consolidation or opacity, or (3) cavitation. The clinical findings had to meet at least one of the following criteria: (1) fever $\left(>38{ }^{\circ} \mathrm{C}\right)$ with no other recognized cause and (2) leukopenia $\left(<4000 / \mathrm{mm}^{3}\right)$ or leukocytosis $\left(\geq 12,000 / \mathrm{mm}^{3}\right)$.
Furthermore, either positive bacterial cultures from sputum, blood, or pleural fluid were required or at least two of the following criteria had to be met: (1) new onset of purulent sputum, change in character of sputum, increased respiratory secretions, or increased suctioning requirement; (2) new onset of worsening cough, dyspnea, or tachypnea; (3) rales or rhonchi; (4) worsening gas exchange. The severity of pneumonia was rated according to the ClavienDindo classification [12].

\section{Endpoints}

The incidence of postoperative pneumonia (Clavien-Dindo classification, grade II or higher) within 90 days after operation was evaluated as the primary endpoint of this study.

Secondary endpoints were postoperative length of hospital stay, postoperative complications (other than pneumonia), and the rates of abnormal results on screening tests.

\section{Study design and statistical analysis}

In this phase II trial, 85 patients were required to provide $80 \%$ power to test the null hypothesis regarding a primary endpoint with an expected value of $3.5 \%$ and a threshold of $7.8 \%$, with one-side testing at the $20 \%$ significance level. The expected value was decided according to the postoperative outcome of 193 patients matching the eligibility criteria of the present study at Shizuoka Cancer Center from January 2010 to May 2012; the incidence of postoperative complications among these patients was $7.8 \%$. 
All statistical analyses were performed using JMP statistical software (version 8.0; SAS Institute, Cary, NC, USA).

\section{Results}

\section{Patients' characteristics}

From May 2015 to August 2016, 86 patients consented to participate and were enrolled in this phase II study. The patients' characteristics are summarized in Table 3 . The mean age was 78 years (range 75-90), and about three quarters were male. The gastric cancer was clinical stage I in 59 of $86(68.5 \%)$ of patients.

Table 3 Characteristics of elderly patients with gastric cancer

\begin{tabular}{ll}
\hline Variables & $n(\%)$ or median (range) \\
\hline Age & $78(75-90)$ \\
Sex & \\
Male & $66(76.7 \%)$ \\
Female & $20(23.3 \%)$ \\
BMI & $22.1(16.1-29.7)$ \\
Tumor size (mm) & $41.5(7-160)$ \\
cStage & \\
I & $59(68.5 \%)$ \\
II & $9(10.5 \%)$ \\
III & $9(10.5 \%)$ \\
IV & $9(10.5 \%)$ \\
PS & \\
0 & $68(79.1 \%)$ \\
1 & $18(20.9 \%)$ \\
Comorbidities & \\
Obstructive ventilatory impairment & $20(23.5 \%)$ \\
Restrictive ventilatory impairment & $10(22.6 \%)$ \\
Combined ventilatory impairment & $4(4.7 \%)$ \\
Diabetes mellitus & $16(18.6 \%)$ \\
Cardiac disease & $16(18.6 \%)$ \\
Smoking history (current or ever) & $59(68.6 \%)$ \\
Preoperative data & \\
White blood cell (/mm $\left.{ }^{3}\right)$ & $5855(3240-10860)$ \\
Hemoglobin (g/dl) & $13.0(8.6-15.9)$ \\
Platelet (/mm $\left.{ }^{3}\right)$ & $204500(75000-426000)$ \\
Asparate aminotransferase (IU/l) & $23(12-60)$ \\
Alanine transaminase (IU/l) & $16.5(7-59)$ \\
Alkaline phosphatase (IU/l) & $4.05(2.5-4.9)$ \\
Total protein (g/dl) & $7.0(4.8-8.0)$ \\
\hline
\end{tabular}

\section{Operative details and pathologic data}

Distal gastrectomy was the most frequently performed procedure, while noncurative operations were performed in four patients $(4.7 \%)$. Regarding the operative approach, the number of patients who underwent open, laparoscopic, and robotic surgery was $31(36.0 \%), 42(48.8 \%)$, and 13 (15.1\%), respectively (Table 4). On pathologic staging, more than half $(52.3 \%)$ of the enrolled patients were diagnosed with stage I cancer, while ten patients were diagnosed with stage IV disease.

\section{Primary endpoint}

Three patients were diagnosed with postoperative pneumonia within 90 days after operation. All three cases were classified as grade II by the Clavien-Dindo classification, and the cause of pneumonia was considered to be aspiration in all three cases. This was an incidence of $3.5 \%$, with a $60 \%$ confidential interval of $1.8-6.3 \%$. This upper limit was lower than the prespecified threshold rate $(7.8 \%)$.

Of three patients with pneumonia, one patient suffered from ileus, and pneumonia was followed by vomiting; however, pneumonia was not followed by vomiting in the other two patients.

The incidence of postoperative pneumonia in patients who underwent TG and DG was $4.8 \%$ (1/21) and 3.1\% (2/ $65)$, respectively, and the difference between operative procedures was not statistically significant $(p=0.72)$.

Table 4 Operative details and pathologic findings

\begin{tabular}{lc}
\hline Variables & $n(\%)$ or median (range) \\
\hline Type of operation & $16(18.6 \%)$ \\
Total gastrectomy & $61(70.9 \%)$ \\
Distal gastrectomy & $5(5.8 \%)$ \\
Proximal gastrectomy & $4(4.7 \%)$ \\
Other & \\
Approach & $31(36.1 \%)$ \\
Open & $42(48.8 \%)$ \\
Laparoscopic surgery & $13(15.1 \%)$ \\
Robot surgery & $295(82-549)$ \\
Operation time (min) & $32.5(0-1267)$ \\
Estimated blood loss (ml) & \\
pStage & $45(52.3 \%)$ \\
I & $14(16.3 \%)$ \\
II & $10(11.7 \%)$ \\
III & $10(11.7 \%)$ \\
IV &
\end{tabular}




\section{Secondary endpoints (hospital stay and other complications)}

Median postoperative hospital stay was 10 days (range 0-62 days).

Postoperative complications other than pneumonia are summarized in Table 5. Pancreatic fistula was the most frequently observed complication, but most cases were grade I to II. Grade IIIa complications were observed in three patients $(3.5 \%)$.

Among five patients with ileus, reflux vomiting was observed in two patients $(2.3 \%)$.

\section{Secondary endpoints (screening tests)}

On screening, 23 patients (26.7\%) had positive results on any of the three tests. Of these 23 patients, 19, 3, and 1 had abnormal results by the symptom questionnaire, MWST, and RSST, respectively. On the symptom questionnaire, eight patients responded "frequently" to question 2 and two each to questions 8, 9, and 10 (Table 1). Postoperative pneumonia was not observed in any of the patients who had abnormal screening tests.

Among 23 patients who screened positive, 20 had rehabilitation medicine division consultations as well as 1 patient with normal screening results who was mistakenly referred to the rehabilitation division. Among these 21 patients, VFSS was performed in 20, while 1 patient mistakenly did not undergo VFSS. Two patients had an abnormal VFSS and underwent intensive individualized rehabilitation (Fig. 1).

\section{Risk factors for postoperative pneumonia}

We performed post hoc analyses to evaluate the preoperative risk factors for postoperative pneumonia. Results showed that none of the preoperative factors were statistically significant risk factors (Supplementary Table 1).

Table 5 Postoperative complications in elderly patients after surgery for gastric cancer

\begin{tabular}{llll}
\hline Complications & Grade I & Grade II & Grade IIIa \\
\hline Pneumonia & 0 & $3(3.5 \%)$ & 0 \\
Pancreatic fistula & $5(5.8 \%)$ & $2(2.1 \%)$ & $1(1.2 \%)$ \\
Bleeding & $1(1.2 \%)$ & 0 & 0 \\
Abdominal abscess & 0 & $4(4.7 \%)$ & $3(3.5 \%)$ \\
Anastomotic leakage & 0 & 0 & $1(1.2 \%)$ \\
Dumping syndrome & 0 & $2(2.1 \%)$ & 0 \\
Ileus & $1(1.2 \%)$ & $4(4.7 \%)$ & 0 \\
Pleural effusion & $5(5.8 \%)$ & 0 & $1(1.2 \%)$ \\
Wound infection & $1(1.2 \%)$ & $1(1.2 \%)$ & 0 \\
\hline
\end{tabular}

Some patients had more than one complication

\section{Discussion}

This phase II study showed that the swallow ability screening tests and pneumonia prevention program were effective in terms of reducing the incidence of postoperative pneumonia in elderly gastric cancer patients who underwent gastrectomy. The incidence of postoperative pneumonia was $3.5 \%$ ( 3 of 86 patients), while the upper limit of the $60 \%$ confidence interval was $6.3 \%$, lower than the threshold of $7.8 \%$; thus, the null hypothesis was rejected.

In the present study, the length of hospital stay and the incidence and severity of complications were acceptable (Table 5) [13]. Most complications were ClavienDindo grade II and were managed medically. Although three patients $(3.5 \%)$ developed grade IIIa abdominal abscesses and required intervention under local anesthesia, grade IIIb or more complications were not observed in any of the patients.

The prevention of ventilator-associated pneumonia has been well investigated, and guidelines have been established $[14,15]$. However, there have been no guidelines on preventing nonventilator-associated postoperative pneumonia. In our hospital, stretching of the neck muscles and simple respiratory training with incentive spirometry were used to prevent postoperative pneumonia before 2015 . However, the incidence of postoperative pneumonia was much higher in elderly patients ( $\geq 75$ years) than in younger patients $(7.8$ vs. $2.7 \%, p<0.01)$ [8]. This is why we established a new program specifically designed to prevent postoperative pneumonia in elderly patients and tested it in this study in those undergoing surgery for gastric cancer.

We should note that postoperative pneumonia was not observed in any of the patients who screened positive for initial swallowing screening tests. Most of these patients were referred to rehabilitation medicine. Intervention by this division is therefore important for reducing the incidence of pneumonia. The only cases of postoperative pneumonia occurred in three patients, none of whom screened positive for swallowing dysfunction. Aspiration was suspected to be the cause of pneumonia in all cases. This finding implies that the screening tests we used were not sufficient to identify patients at high risk of aspiration pneumonia. Because it is not practical to perform VFSS for all elderly patients, more accurate screening tests are needed to identify patients with abnormal swallow ability.

For this study, we adopted three types of easily performed screening tests but found very few patients whose MSWT or RSST was abnormal. Most patients suspected to have abnormal swallowing ability were identified by the symptom questionnaire. While a total of 23 patients had 
Fig. 1 Flowchart of elderly patients with gastric cancer screened for swallowing dysfunction, with interventions as indicated to prevent postoperative pneumonia

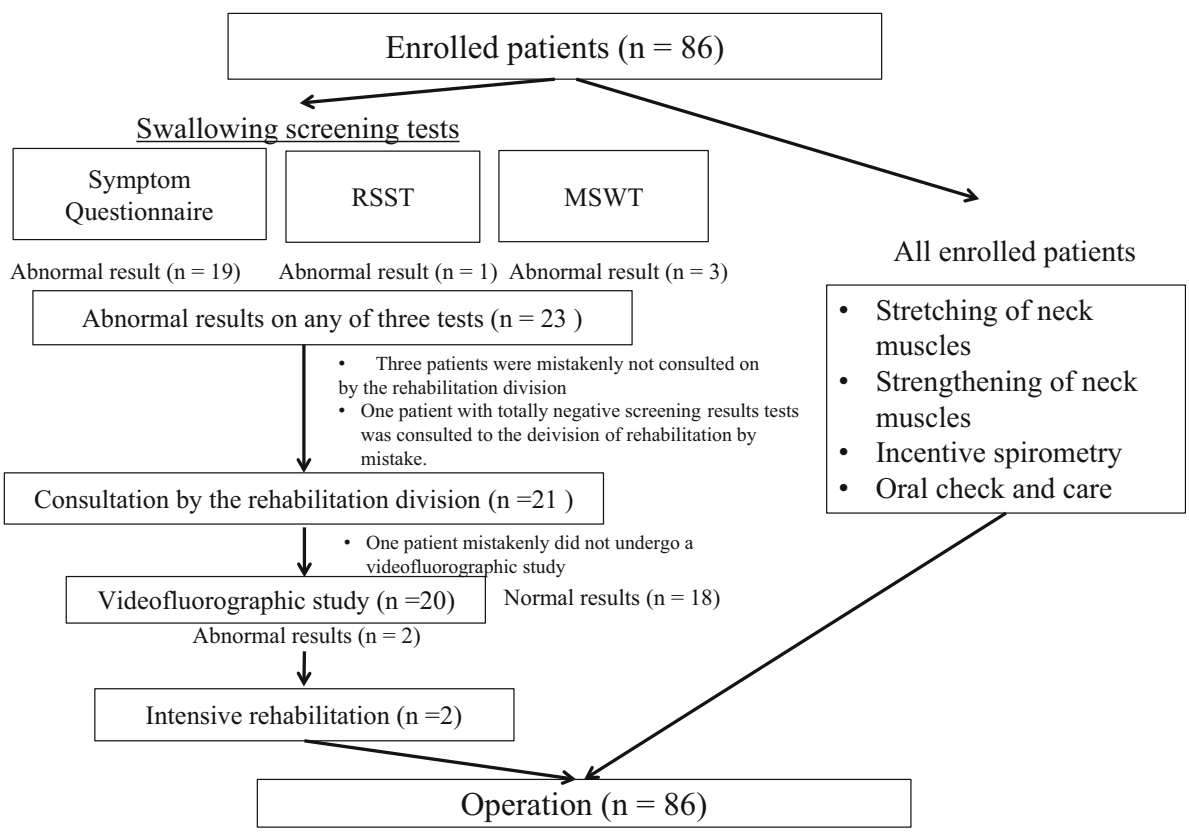

abnormal results on any of the three tests, the only 2 who had an abnormal VFSS screened positive only on the symptom questionnaire, not on the other two tests. This suggests that questionnaire is the most promising screening test. However, among 14 questions in the questionnaire, only questions $2,8,9$, and 10 were noted by more than one patient to occur "frequently." This means that the questionnaire could be simplified. However, questions 2,8 , and 9 do not necessarily indicate a specific swallowing problem, as gastric cancer alone might account for these symptoms in patients whose swallow ability is normal. Thus, we consider that the content of the questionnaire should be amended for elderly patients with gastric cancer.

None of the strategies included in this study were new. The screening tests were introduced in the guidelines for deglutition disorders published by the Oto-Rhino-Laryngological Society of Japan. The importance of oral care has been proven in patients undergoing esophagectomy [16]. Suprahyoid muscle strengthening has been shown to be effective in restoring oral feeding in patients with deglutitive failure. However, the combination of these measures has not previously been evaluated. To the best of our knowledge, this is the first study to show the significance of a team approach, including surgeons, rehabilitation medicine physicians, dentists, nurses, physical therapists, and speech-hearinglanguage therapists. This approach is simple and easy, so it can be used in hospitals anywhere in the world.

There are several limitations of this study. First, this study had low $\alpha$ values and a small patient number. Second, this study was conducted at a single center. Thus, a prospective multicenter phase III study with a larger number of patients is needed for more definitive results and more universal usage of these programs. Finally, as previously described, the screening tests we adopted were not successful. We consider that they should be amended for future studies. Concretely, MSWT and RSST are not necessary, and a more simplified questionnaire (question numbers 2, 8, 9, and 10) may be sufficient in the future. Nevertheless, it is noteworthy that perioperative intensive rehabilitation could prevent the occurrence of postoperative pneumonia in two patients whose VFSS was abnormal.

In conclusion, although the screening tests we adopted here were not sufficient to identify patients at high risk of aspiration pneumonia, perioperative interventions using a team approach might be effective in reducing the incidence of postoperative pneumonia in elderly patients with gastric cancer.

\section{Compliance with ethical standards}

Human rights statement All procedures followed were in accordance with the ethical standards of the responsible committee on human experimentation (institutional and national) and with the Helsinki Declaration of 1964 and later versions.

Informed consent Informed consent was obtained from all patients for being included in the study.

Funding This study was funded by the National Cancer Center Research and Development Fund, 26-A-4 Scientific research on multi-institutional trials to establish new standard treatment of solid tumors in adults, and Practical Research for Innovative Cancer Control from the Japan Agency for Medical Research and Development, AMED (15ck0106043h0002).

Conflict of interest The authors declare that they have no conflict of interest. 


\section{References}

1. Hundahl SA, Menck HR, Mansour EG, Winchester DP. The national cancer data base report on gastric carcinoma. Cancer. 1997;80(12):2333-41.

2. Pisani P, Parkin DM, Bray F, Ferlay J. Estimates of the worldwide mortality from 25 cancers in 1990. Int J Cancer. 1999;83(1):18-29.

3. Gretschel S, Estevez Schwarz L, Hünerbein M, Schneider U, Schlag P. Gastric cancer surgery in elderly patients. World J Surg. 2006;30(8):1468-74.

4. Saif MW, Makrilia N, Zalonis A, Merikas M, Syrigos K. Gastric cancer in the elderly: an overview. Eur J Surg Oncol. 2010;36(8):709-17.

5. Kunisaki C, Akiyama H, Nomura M, Matsuda G, Otsuka Y, Ono $\mathrm{H}$, et al. Comparison of surgical outcomes of gastric cancer in elderly and middle-aged patients. Am J Surg. 2006;191(2):216-24.

6. Wu CW, Lo SS, Shen KH, Hsieh MC, Lui WY, P'Eng FK. Surgical mortality, survival, and quality of life after resection for gastric cancer in the elderly. World J Surg. 2000;24(4):465-72.

7. Yamada H, Shinohara T, Takeshita M, Umesaki T, Fujimori Y, Yamagishi K. Postoperative complications in the oldest old gastric cancer patients. Int J Surg. 2013;11(6):467-71.

8. Miki Y, Makuuchi R, Tokunaga M, Tanizawa Y, Bando E, Kawamura $\mathrm{T}$, et al. Risk factors for postoperative pneumonia after gastrectomy for gastric cancer. Surg Today. 2016;46(5): $552-6$.
9. The Oto-Rhino-Laryngological Society of Japan. The treatment guideline of deglutition disorders. 2nd ed. Kanehara Shuppan; 2012.

10. Wu MC, Chang YC, Wang TG, Lin LC. Evaluating swallowing dysfunction using a 100-ml water swallowing test. Dysphagia. 2004;19(1):43-7.

11. Shaker R, Easterling C, Kern M, Nitschke T, Massey B, Daniels $\mathrm{S}$, et al. Rehabilitation of swallowing by exercise in tube-fed patients with pharyngeal dysphagia secondary to abnormal UES opening. Gastroenterology. 2002;122(5):1314-21.

12. Clavien PA, Barkun J, de Oliveira ML, Vauthey JN, Dindo D, Schulick RD, et al. The Clavien-Dindo classification of surgical complications: 5-year experience. Ann Surg. 2009;250(2):187-96.

13. Sugisawa N, Tokunaga M, Makuuchi R, Miki Y, Tanizawa Y, Bando E, et al. A phase II study of an enhanced recovery after surgery protocol in gastric cancer surgery. Gastric Cancer. 2016;19(3):961-7.

14. Hunter JD. Ventilator associated pneumonia. BMJ. 2012;344:e3325.

15. Rea-Neto A, Youssef NC, Tuche F, Brunkhorst F, Ranieri VM, Reinhart K, et al. Diagnosis of ventilator-associated pneumonia: a systematic review of the literature. Crit Care. 2008;12(2):R56.

16. Akutsu Y, Matsubara H, Shuto K, Shiratori T, Uesato M, Miyazawa Y, et al. Pre-operative dental brushing can reduce the risk of postoperative pneumonia in esophageal cancer patients. Surgery. 2010;147(4):497-502. 